\title{
Error Analysis and Error Allocation for Turntable Systems Used in GyroWheel Calibration Tests
}

\author{
Yuyu Zhao, Hui Zhao, Xin Huo, and Yu Yao \\ Control and Simulation Center, Harbin Institute of Technology, Harbin 150001, China \\ Correspondence should be addressed to Yu Yao; yaoyu_academic@163.com
}

Received 11 April 2017; Revised 12 September 2017; Accepted 28 September 2017; Published 28 November 2017

Academic Editor: Paul Williams

Copyright (c) 2017 Yuyu Zhao et al. This is an open access article distributed under the Creative Commons Attribution License, which permits unrestricted use, distribution, and reproduction in any medium, provided the original work is properly cited.

\begin{abstract}
Calibration tests are of great importance to ensure rate-sensing accuracy of GyroWheel, an innovative attitude determination and control device. In the process of calibration tests, turntable errors are inevitable, which hinder the calibration accuracy and ratesensing capability. Hence, error analysis for GyroWheel calibration tests is conducted, and the relationship between the calibration accuracy and the orientation error is established based on analytical derivation and numerical simulations. Subsequently, an error model of the turntable system is derived using rigid body kinematics, by which the relationship between the orientation error and turntable errors is described. According to sensitivity analysis and manufacturing capability, an error allocation method is proposed to determine the accuracy requirement of the test turntable, and the effectiveness of the proposed method is verified by repeated simulation tests. Based on the presented analysis and proposed method in this paper, the effects of various turntable errors on the calibration accuracy can be obtained quantitatively, and a theoretical basis for the determination of the turntable accuracy is provided, which are of great significance to guide the calibration tests and improve the calibration accuracy of GyroWheel.
\end{abstract}

\section{Introduction}

The development of small spacecrafts has received a lot of attention in recent years [1,2]. As GyroWheel is an innovative attitude determination and control device, it offers the potential to meet the performance, mass, and cost requirements of small spacecrafts. It provides control torques about three axes while also measuring the spacecraft angular rates about the two axes perpendicular to the spin direction, which improves the integration and efficiency of attitude control system in small spacecrafts $[3,4]$.

The conception of GyroWheel is inspired by a dynamically tuned gyroscope (DTG). However, it has a larger rotor and tilt angles, as well as a time-varying spinning rate due to its multifunction capability. When the GyroWheel is used to measure angular rates, erroneous torques, which are often caused by design limitation and constructional deficiencies, act on the rotor of the GyroWheel. These imperfections give rise to precession of the rotor, resulting in measurement errors of angular rates [5-7].

To maintain high-accuracy measurement, calibration tests are of great importance in the application of the GyroWheel. The goal of calibration tests is to fully characterize the outputs of the inertial instruments so that a nonideal behavior can be modelled and compensated [5, 7-10]. Generally, calibration tests are carried out using a turntable to provide the intended orientation of the GyroWheel. Turntable errors, including position error, wobble error, orthogonality error, and intersection error, will inevitably affect the GyroWheel system in calibration tests [11]. Although the effects of turntable errors can be reduced by using a high-accuracy turntable, the costs of calibration tests will increase significantly. To reconcile the requirement of calibration accuracy and test costs, 
the effects of turntable errors on calibration accuracy should be analyzed, and error allocation methods for turntable system should be investigated in consideration of the sensitivities of these errors and the manufacturing capability in practical engineering before calibration tests are carried out.

Generally, error models of multi-DOF motion systems, such as machine tools and turntables, can be derived using rigid body kinematics [11-14]. Additionally, a few studies about error effect analysis for calibration tests have been conducted. An error analysis of precision centrifuge, an equipment used in calibration tests, was conducted, and its effect on the calibration accuracy of gyro accelerometers was discussed in $[15,16]$. The effect of position error on calibration accuracy of inertial instruments has been analyzed in $[17,18]$. As mentioned in [19], the models of a turntable's orthogonality error and horizontality error were established, and quantitative analyses of their effects on calibration accuracy were presented. However, the existing studies have mainly analyzed a single error component's effect on calibration accuracy, in which other error factors are not considered; obviously, this is not consistent with the actual situation. To the authors' knowledge, little attention has been focused on the error allocation problem for the multi-DOF motion systems. By assuming that each of the errors contributes equally to the overall error, the error allocation problem for a 6-DOF parallel manipulator was discussed in [20, 21]. However, the assumption of equivalent effects of the errors is inappropriate for the turntable system. Although orthogonal experimental design has been used to determine the significant level of each error factor for a 3 DOF robotic mechanism [22], it is difficult to obtain a quantitative error allocation result. There were no relevant studies on the error allocation method for the turntable system used in calibration tests.

Motivated by these facts, the error analysis and error allocation problems of the turntable used in GyroWheel's calibration tests are investigated in this paper. According to the multiposition calibration theory and rate-sensing model of the GyroWheel, error analysis for GyroWheel calibration tests is conducted quantitatively. With numerical simulations, the relationships between the ratesensing errors of the GyroWheel and the orientation errors are obtained. The orientation error due to manufacture imperfections and design limitations of the turntable system is modelled based on rigid body kinematics, with consideration of the interaction of these turntable errors. Then an error allocation method for the turntable system based on sensitivity analysis is proposed, and the effectiveness of the proposed method is verified by repeated simulation tests.

The remainder of this paper is as follows. In Section 2, the GyroWheel calibration theory is described, and the effect of orientation error on the calibration accuracy is analyzed. In Section 3, the error model of a two-axis turntable system is established based on rigid body kinematics, which develops a relationship between the turntable error components and the orientation error of the GyroWheel. In Section 4, sensitivities of the orientation error to the

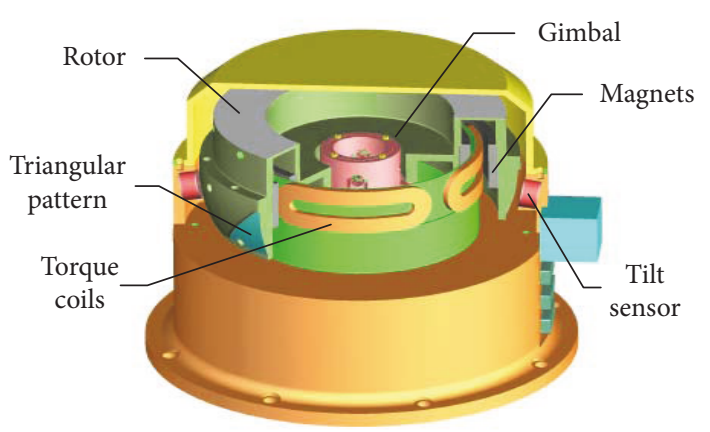

FIgURE 1: Cutaway view of a GyroWheel.

individual error components and the manufacturing capability are discussed. On this basis, an error allocation method is given to determine the accuracy requirement of the test turntable. And several concluding remarks are given in Section 5.

\section{GyroWheel Calibration Theory and Error Analysis for Calibration Tests}

2.1. Overview of GyroWheel Calibration Theory. The GyroWheel is an innovative attitude determination and control instrument. A cutaway isometric view of the GyroWheel is shown in Figure 1. When the GyroWheel is used to measure angular rates, erroneous torques give rise to precession of the rotor resulting in drift errors. In the null tilt condition, the rate-sensing equations of the GyroWheel are given in the following equation, with consideration of the drift errors [5]:

$$
\begin{aligned}
\omega_{x}= & k_{y} i_{y}-D(x)_{F}-D(x)_{x} a_{x}-D(x)_{y} a_{y}-D(x)_{z} a_{z} \\
& -D(x)_{x x} a_{x}^{2}-D(x)_{z z} a_{z}^{2}-D(x)_{x y} a_{x} a_{y}, \\
\omega_{y}= & k_{x} i_{x}-D(y)_{F}-D(y)_{x} a_{x}-D(y)_{y} a_{y}-D(y)_{z} a_{z} \\
& -D(y)_{y y} a_{y}^{2}-D(y)_{z z} a_{z}^{2}-D(y)_{y x} a_{x} a_{y},
\end{aligned}
$$

where $D(x), D(y)$ represent the drift errors of $x, y$ axes, $D$ $(x)_{F}, D(y)_{F}$ are $g$ insensitive terms, $D(x)_{i}, D(y)_{i}, i=x, y, z$, are $g$ sensitive error coefficients, and $D(x)_{i j}, i j=x x, z z, x y$, $D(y)_{i j}, i j=y y, z z, y x$, are $g^{2}$ sensitive error coefficients. $a_{x}$, $a_{y}, a_{z}$ represent acceleration components of the gravity vector, $\omega_{x}, \omega_{y}$ are external angular velocities, $k_{x}, k_{y}$ are torque scale factors of the GyroWheel, and $i_{x}, i_{y}$ are the currents in the torque coils.

In an effort to improve the rate-sensing accuracy of the GyroWheel, multiposition tests are performed to calibrate the GyroWheel. Multiposition tests make use of a two-axis turntable to provide the intended orientation for the GyroWheel. A schematic representation of a turntable is shown in Figure 2. The earth's rotation rate and gravitational acceleration are regarded as the nominal inputs 

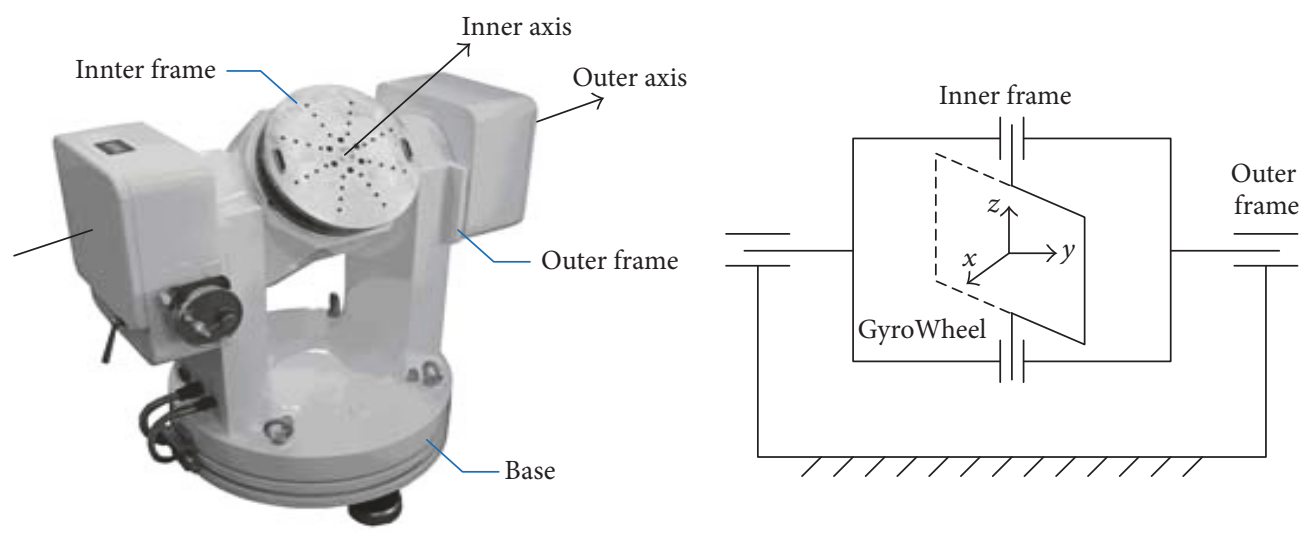

FIGURE 2: Schematic representation of a two-axis turntable.

of the GyroWheel. At each position, an equation expressed as (1) can be obtained using the inputs and outputs of the GyroWheel. Therefore, the error coefficients can be calculated when enough tests of different positions are conducted.

Take the $x$-axis model of the GyroWheel as an example. According to the rate-sensing model of the GyroWheel and multiposition calibration theory [10], we have

$$
\begin{gathered}
{\left[\begin{array}{c}
k_{y} i_{y 1} \\
k_{y} i_{y 2} \\
\vdots \\
k_{y} i_{y n}
\end{array}\right]-\left[\begin{array}{c}
\omega_{x 1} \\
\omega_{x 2} \\
\vdots \\
\omega_{x n}
\end{array}\right]} \\
=\left[\begin{array}{ccccccc}
1 & a_{x 1} & a_{y 1} & a_{z 1} & a_{x 1}^{2} & a_{z 1}^{2} & a_{x 1} a_{y 1} \\
1 & a_{x 2} & a_{y 2} & a_{z 2} & a_{x 2}^{2} & a_{z 2}^{2} & a_{x 2} a_{y 2} \\
\vdots & \vdots & \vdots & \vdots & \vdots & \vdots & \vdots \\
1 & a_{x n} & a_{y n} & a_{z n} & a_{x n}^{2} & a_{z n}^{2} & a_{x n} a_{y n}
\end{array}\right]\left[\begin{array}{c}
D(x)_{F} \\
D(x)_{x} \\
D(x)_{y} \\
D(x)_{z} \\
D(x)_{x x} \\
D(x)_{z z} \\
D(x)_{x y}
\end{array}\right],
\end{gathered}
$$

where $n$ is the number of test positions, $i_{y j}, j=1,2, \ldots, n$, represent the currents in the torque coils, $\omega_{x i}, i=1,2, \ldots, n$, represent the earth rate components of each test position, and $a_{x i}, a_{y i}, a_{z i}, i=1,2, \ldots, n$, represent the gravitational acceleration components of each test position.

The initial orientation of the GyroWheel axes is northwest-up. Define that $\omega_{e}$ is the earth's rotation rate, $\lambda$ is the latitude, $\phi_{1 i}, \phi_{2 i}$ are the rotation angles of the turntable, and the subscript $i$ means the $i$ th test position. The gravitational acceleration components and the earth rate components can be expressed as follows:

$$
\begin{aligned}
& a_{x i}=-\sin \phi_{1 i} \cos \phi_{2 i}, \\
& a_{y i}=\sin \phi_{1 i} \sin \phi_{2 i}, \\
& a_{z i}=\cos \phi_{1 i}, \\
& \omega_{x i}=\omega_{e} \cos \phi_{2 i}\left(\cos \phi_{1 i} \cos \lambda-\sin \phi_{1 i} \sin \lambda\right), \\
& \omega_{y i}=\omega_{e} \sin \phi_{2 i}\left(-\cos \phi_{1 i} \cos \lambda+\sin \phi_{1 i} \sin \lambda\right), \\
& \omega_{z i}=\omega_{e}\left(\sin \phi_{1 i} \cos \lambda+\cos \phi_{1 i} \sin \lambda\right) .
\end{aligned}
$$

Denote

$$
\begin{aligned}
& \mathbf{b}_{1}=\left[\begin{array}{c}
k_{y} i_{y 1} \\
k_{y} i_{y 2} \\
\vdots \\
k_{y} i_{y n}
\end{array}\right] \\
& \mathbf{b}_{2}=\left[\begin{array}{c}
\omega_{x 1} \\
\omega_{x 2} \\
\vdots \\
\omega_{x n}
\end{array}\right] \\
& \mathbf{A}=\left[\begin{array}{ccccccc}
1 & a_{x 1} & a_{y 1} & a_{z 1} & a_{x 1}^{2} & a_{z 1}^{2} & a_{x 1} a_{y 1} \\
1 & a_{x 2} & a_{y 2} & a_{z 2} & a_{x 2}^{2} & a_{z 2}^{2} & a_{x 2} a_{y 2} \\
\vdots & \vdots & \vdots & \vdots & \vdots & \vdots & \vdots \\
1 & a_{x n} & a_{y n} & a_{z n} & a_{x n}^{2} & a_{z n}^{2} & a_{x n} a_{y n}
\end{array}\right],
\end{aligned}
$$

$$
\boldsymbol{\beta}=\left[\begin{array}{c}
D(x)_{F} \\
D(x)_{x} \\
D(x)_{y} \\
D(x)_{z} \\
D(x)_{x x} \\
D(x)_{z z} \\
D(x)_{x y}
\end{array}\right] .
$$




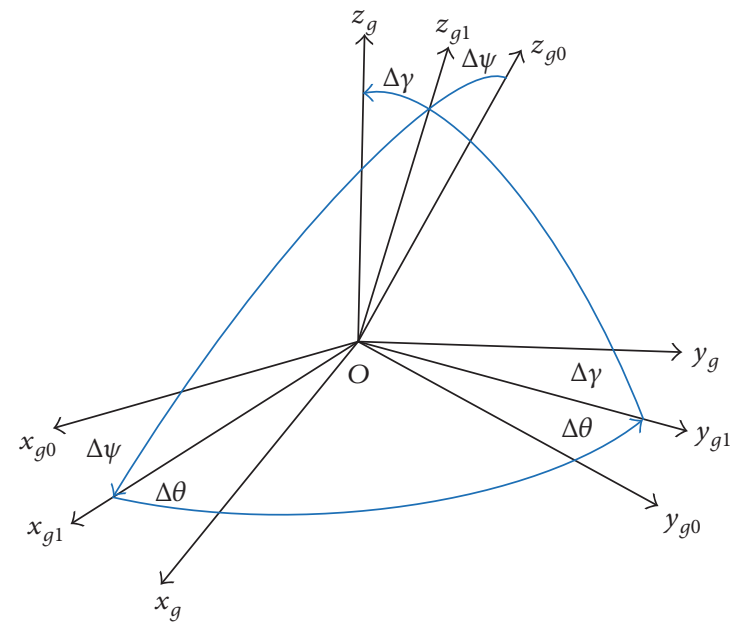

FIGURE 3: Orientation error of GyroWheel axes. $O x_{g 0} y_{g 0} z_{g 0}$ : the intended orientation of the GyroWheel axes. Ox $x_{g} z_{g}$ : the actual orientation of the GyroWheel axes.

Then (2) can be rewritten as

$$
\mathbf{b}_{1}-\mathbf{b}_{2}=\mathbf{A} \beta
$$

The calibration coefficient vector $\boldsymbol{\beta}$ can be solved utilizing the method of least squares:

$$
\widehat{\boldsymbol{\beta}}_{1}=\left(\mathbf{A}^{T} \mathbf{A}\right)^{-1} \mathbf{A}^{T}\left(\mathbf{b}_{1}-\mathbf{b}_{2}\right)
$$

2.2. Error Analysis for GyroWheel Calibration Tests. Due to manufacture imperfections and design limitations of the turntable system, the orientation of the GyroWheel axes will be deviated from the intended one. As a result, there are deviations between the actual earth rate components and the ideal earth rate components, as well as the gravitational acceleration components. Use $\Delta \psi, \Delta \theta$, $\Delta \gamma$ to denote rotational errors about $y$-, $z$-, and $x$-axes, respectively (Figure 3 ).

With the assumption of small angular errors, the transformation matrix describing the orientation error of the GyroWheel with respect to its ideal orientation is given below:

$$
\Delta \mathbf{R}=\left[\begin{array}{ccc}
1 & -\Delta \theta & \Delta \psi \\
\Delta \theta & 1 & -\Delta \gamma \\
-\Delta \psi & \Delta \gamma & 1
\end{array}\right]
$$

The actual earth rate components and gravitational acceleration components can be calculated as follows:

$$
\begin{aligned}
{\left[\begin{array}{lll}
\omega_{x i}^{\prime} & \omega_{y i}^{\prime} & \omega_{z i}^{\prime}
\end{array}\right] } & =\left[\begin{array}{lll}
\omega_{x i} & \omega_{y i} & \omega_{z i}
\end{array}\right] \Delta \mathbf{R}, \\
{\left[\begin{array}{lll}
a_{x i}^{\prime} & a_{y i}^{\prime} & a_{z i}^{\prime}
\end{array}\right] } & =\left[\begin{array}{lll}
a_{x i} & a_{y i} & a_{z i}
\end{array}\right] \Delta \mathbf{R} .
\end{aligned}
$$

In the presence of the orientation error, the regression model of multiposition calibration is given by

$$
\mathbf{b}_{1}-\mathbf{b}_{2}^{\prime}=\mathbf{A}^{\prime} \boldsymbol{\beta},
$$

where

$$
\begin{aligned}
\mathbf{b}_{2}^{\prime} & =\left[\begin{array}{c}
\omega_{x 1}^{\prime} \\
\omega_{x 2}^{\prime} \\
\vdots \\
\omega_{x n}^{\prime}
\end{array}\right], \\
\mathbf{A}^{\prime} & =\left[\begin{array}{ccccccc}
1 & a_{x 1}^{\prime} & a_{y 1}^{\prime} & a_{z 1}^{\prime} & a_{x 1}^{\prime 2} & a_{z 1}^{\prime 2} & a_{x 1}^{\prime} a_{y 1}^{\prime} \\
1 & a_{x 2}^{\prime} & a_{y 2}^{\prime} & a_{z 2}^{\prime} & a_{x 2}^{\prime 2} & a_{z 2}^{\prime 2} & a_{x 2}^{\prime} a_{y 2}^{\prime} \\
\vdots & \vdots & \vdots & \vdots & \vdots & \vdots & \vdots \\
1 & a_{x n}^{\prime} & a_{y n}^{\prime} & a_{z n}^{\prime} & a_{x n}^{\prime 2} & a_{z n}^{\prime 2} & a_{x n}^{\prime} a_{y n}^{\prime}
\end{array}\right] .
\end{aligned}
$$

Then the true value of the calibration coefficient vector can be calculated as follows:

$$
\widehat{\boldsymbol{\beta}}_{2}=\left(\mathbf{A}^{\prime T} \mathbf{A}^{\prime}\right)^{-1} \mathbf{A}^{\prime T}\left(\mathbf{b}_{1}-\mathbf{b}_{2}^{\prime}\right)
$$

The actual calibration results are given by (6), and the deviation of the calibration results caused by the orientation error can be expressed as

$$
\Delta \boldsymbol{\beta}=\widehat{\boldsymbol{\beta}}_{1}-\widehat{\boldsymbol{\beta}}_{2}
$$

The deviation $\Delta \boldsymbol{\beta}$ results in GyroWheel's rate-sensing error $\Delta \omega_{x}$; the rate-sensing error vector at the test positions can be calculated:

$$
\Delta \widehat{\boldsymbol{\omega}}_{x}=\left(\mathbf{b}_{1}-\mathbf{A} \widehat{\boldsymbol{\beta}}_{2}\right)-\mathbf{b}_{2}^{\prime}
$$

Define

$$
\Delta \omega_{x}=\left\|\Delta \widehat{\omega}_{x}\right\|_{\infty}
$$

$\Delta \omega_{x}$ represents the rate-sensing error of $x$-axis. The rate-sensing error of $y$-axis, denoted by $\Delta \omega_{y}$, can be calculated in the same way. Obviously, the rate-sensing errors $\Delta \omega_{x}$ and $\Delta \omega_{y}$ are affected by the orientation error described by $\Delta \psi, \Delta \theta, \Delta \gamma$. If the values of $\Delta \psi, \Delta \theta, \Delta \gamma$ are given, the corresponding values of the rate-sensing errors $\Delta \omega_{x}$ and $\Delta \omega_{y}$ can be calculated by several matrix operations. To facilitate an error analysis, a numerical method is utilized. A series of rate-sensing errors are obtained with numerical simulations when a series of $\Delta \psi, \Delta \theta, \Delta \gamma$ are given. The relationships between the rate-sensing errors $\Delta \omega_{x}, \Delta \omega_{y}$ and the orientation error described by $\Delta \psi, \Delta \theta, \Delta \gamma$ are expressed in a visualized way, as shown 


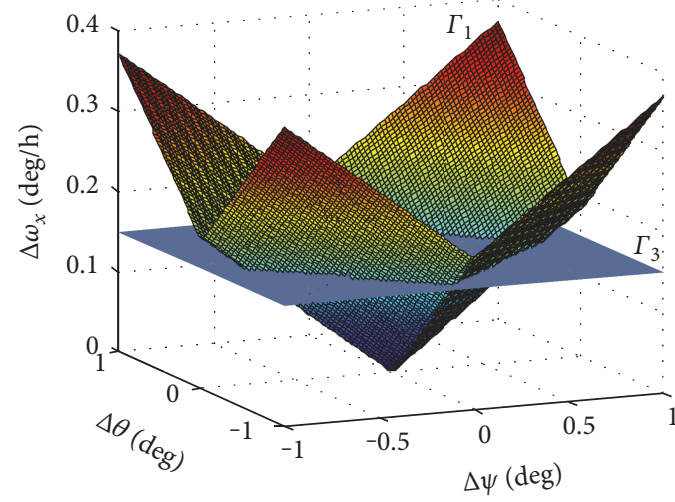

(a) Relationship between $\Delta \omega_{x}$ and $\Delta \psi, \Delta \theta$

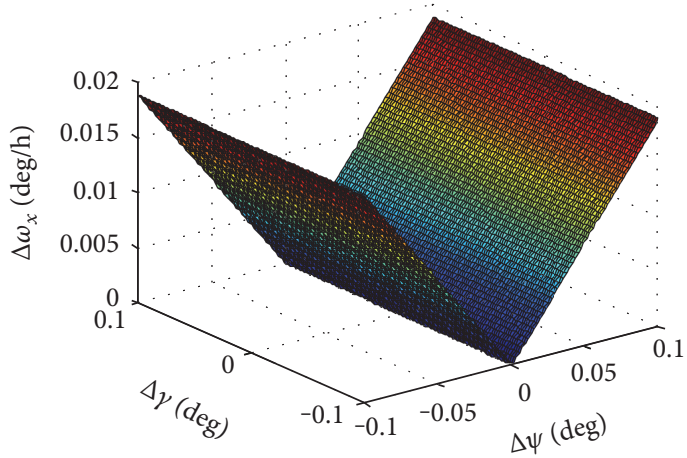

(c) Relationship between $\Delta \omega_{x}$ and $\Delta \psi, \Delta \gamma$

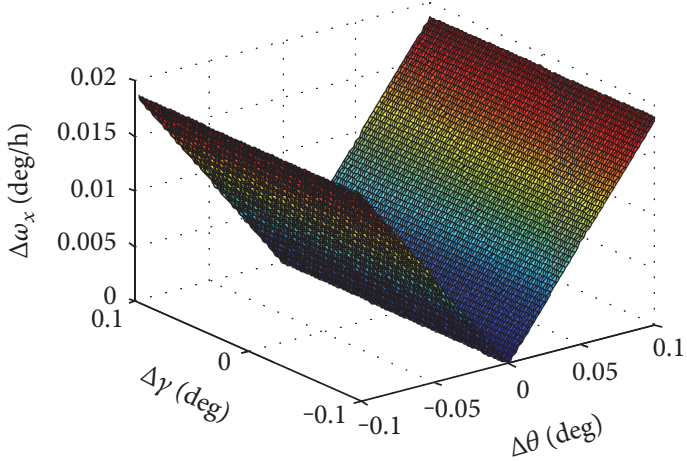

(e) Relationship between $\Delta \omega_{x}$ and $\Delta \theta, \Delta \gamma$

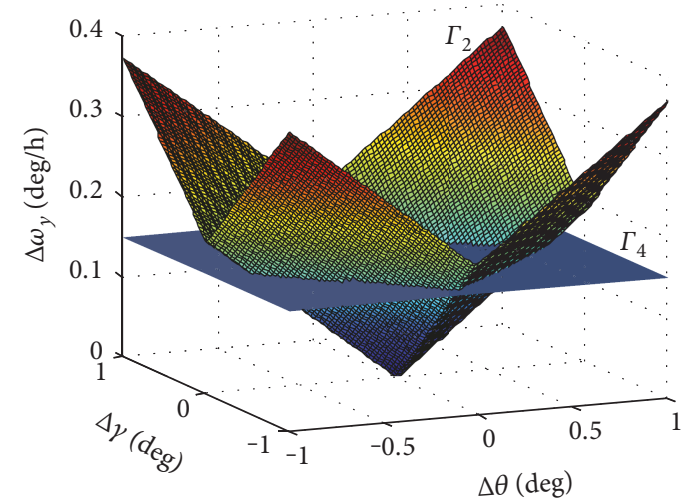

(b) Relationship between $\Delta \omega_{y}$ and $\Delta \theta, \Delta \gamma$

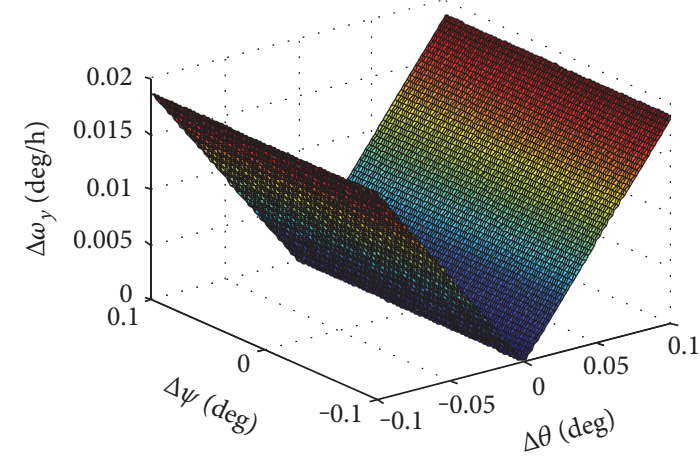

(d) Relationship between $\Delta \omega_{y}$ and $\Delta \theta, \Delta \psi$

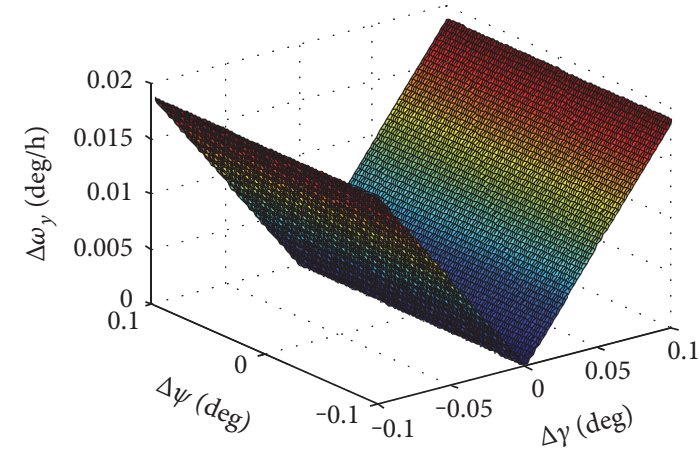

(f) Relationship between $\Delta \omega_{y}$ and $\Delta \gamma, \Delta \psi$

FIGURE 4: Relationships between rate-sensing errors and orientation error.

in Figure 4. The two surfaces describing the relationships in Figures 4(a) and 4(b) are denoted by $\Gamma_{1}, \Gamma_{2}$. From the simulation results, the following conclusions can be made:

(1) As seen in Figures 4(a)-4(f), the rate-sensing error of $x$-axis $\Delta \omega_{x}$ is caused by $\Delta \psi, \Delta \theta$, and it is not affected by $\Delta \gamma$. The rate-sensing error of $y$-axis $\Delta \omega_{y}$ is caused by $\Delta \theta, \Delta \gamma$, and it is not affected by $\Delta \psi$.

(2) As seen in Figure 4(a), the surface $\Gamma_{1}$ is symmetric about the planes $\Delta \psi=0$ and $\Delta \theta=0$. As seen in
Figure 4(b), the surface $\Gamma_{2}$ is symmetric about the planes $\Delta \theta=0$ and $\Delta \gamma=0$. Therefore, $\Delta \omega_{x}$ is an even function of $\Delta \psi, \Delta \theta$, and $\Delta \omega_{y}$ is an even function of $\Delta \theta, \Delta \gamma$.

(3) As seen in Figures 4(a)-4(f), $\Delta \omega_{x}$ rises with the increasing of $|\Delta \psi|,|\Delta \theta|$, and $\Delta \omega_{y}$ rises with the increasing of $|\Delta \theta|,|\Delta \gamma|$.

Use $\omega_{m}$ to denote the required calibration accuracy of the GyroWheel; that is, $\Delta \omega_{x} \leq \omega_{m}, \Delta \omega_{y} \leq \omega_{m}$. By drawing two planes defined by the implicit equations $\Gamma_{3}: \Delta \omega_{x}=\omega_{m}$ 
and $\Gamma_{4}: \Delta \omega_{y}=\omega_{m}$ in Figures 4(a) and 4(b), respectively, two lines of intersection are obtained, and the requirement of the orientation error can be determined easily and is denoted by

$$
\begin{aligned}
& |\Delta \psi| \leq I_{\psi}, \\
& |\Delta \theta| \leq I_{\theta}, \\
& |\Delta \gamma| \leq I_{\gamma} .
\end{aligned}
$$

2.3. Example. A GyroWheel with the required calibration accuracy of $0.01 \%$ is taken as an example. The nominal values of the calibration coefficients are listed in Table 1. According to the above analysis, the line of intersection between $\Gamma_{1}$ and $\Gamma_{3}$, and the line of intersection between $\Gamma_{2}$ and $\Gamma_{4}$ are given in Figure 5 .

As seen in Figure 5, the rectangular areas in black represent the ranges of $\Delta \psi, \Delta \theta, \Delta \gamma$, in which the requirement of the calibration accuracy can be met. To maximize the areas of the two rectangles in Figure 5, the requirement of the orientation error is determined as follows:

$$
\begin{aligned}
& |\Delta \psi| \leq 0.027^{\circ}, \\
& |\Delta \theta| \leq 0.027^{\circ}, \\
& |\Delta \gamma| \leq 0.027^{\circ} .
\end{aligned}
$$

\section{Error Analysis of Turntable System}

3.1. Overview of Turntable Errors. There are four main sources of errors in a turntable system; they are position error, wobble error, orthogonality error, and intersection error.

Position error is defined as the difference between the actual and the intended rotation angles of the turntable's rotating shaft, which is influenced by servo precision and measurement accuracy of the turntable system.

Wobble error can be divided into axial wobble error, radial wobble error, and angular wobble error, as illustrated in Figure 6, where $\Delta s_{a}$ is the axial wobble error, $\Delta s_{r}$ is the radial wobble error, and $\Delta \alpha$ is the angular wobble error. In practical engineering, $\Delta s_{a}$ and $\Delta s_{r}$ are small enough to be ignored; thus, wobble error refers specifically to angular wobble error $\Delta \alpha$ in this paper $[11,23,24]$.

Orthogonality error and intersection error are induced by mechanical imperfections of the turntable structure and misalignment of turntable elements.

For the two-axis turntable given in Figure 2, a summary of the error components involved is shown in Table 2.

3.2. Error Modeling for Turntable System. The error model of the turntable system can be developed based on rigidbody kinematics. The two-axis turntable system, as shown in Figure 2, consists of three bodies: the base, the outer frame, and the inner frame. In addition, the GyroWheel being attached to the inner frame should be considered. In an effort to derive the error model of the turntable, five coordinate systems are introduced. They are the base coordinate system $\mathscr{F}_{0}: O_{0} X_{0} Y_{0} Z_{0}$, the outer axis coordinate system $\mathscr{F}_{1}: O_{1} X_{1} Y_{1} Z_{1}$, the reference coordinate system
TABLE 1: Nominal values of calibration coefficients.

\begin{tabular}{lccc}
\hline$D(x)_{\mathrm{F}}(\mathrm{deg} / \mathrm{h})$ & -93.3 & $D(y)_{F}(\mathrm{deg} / \mathrm{h})$ & 105.3 \\
$D(x)_{x}(\mathrm{deg} / \mathrm{h} / g)$ & -43.2 & $D(y)_{x}(\mathrm{deg} / \mathrm{h} / g)$ & 2.2 \\
$D(x)_{y}(\mathrm{deg} / \mathrm{h} / g)$ & 3.5 & $D(y)_{y}(\mathrm{deg} / \mathrm{h} / g)$ & -33.2 \\
$D(x)_{z}(\mathrm{deg} / \mathrm{h} / g)$ & -1.2 & $D(y)_{z}(\mathrm{deg} / \mathrm{h} / g)$ & -0.1 \\
$D(x)_{x x}\left(\mathrm{deg} / \mathrm{h} / g^{2}\right)$ & 2.1 & $D(y)_{y y}\left(\mathrm{deg} / \mathrm{h} / g^{2}\right)$ & -10.6 \\
$D(x)_{z z}\left(\mathrm{deg} / \mathrm{h} / g^{2}\right)$ & -0.089 & $D(y)_{z z}\left(\mathrm{deg} / \mathrm{h} / g^{2}\right)$ & -10.38 \\
$D(x)_{x y}\left(\mathrm{deg} / \mathrm{h} / g^{2}\right)$ & 17.4 & $D(y)_{x y}\left(\mathrm{deg} / \mathrm{h} / g^{2}\right)$ & 47.8 \\
\hline
\end{tabular}

of the inner axis $\mathscr{F}_{2}: \mathrm{O}_{2} X_{2} Y_{2} Z_{2}$, the inner axis coordinate system $\mathscr{F}_{3}: O_{3} X_{3} Y_{3} Z_{3}$ and the GyroWheel coordinate system $\mathscr{F}_{4}: O_{g} X_{g} Y_{g} Z_{g} . \mathscr{F}_{0}, \mathscr{F}_{1}, \mathscr{F}_{3}$, and $\mathscr{F}_{4}$ are four body-fixed coordinate systems, which are attached to the base, the outer frame, the inner frame, and the GyroWheel, respectively. $\mathscr{F}_{2}$ is an interim coordinate system; the relation between $\mathscr{F}_{2}$ and $\mathscr{F}_{1}$ is determined by the orthogonality error and intersection error of the turntable system. An illustration of these coordinate systems is shown in Figure 7.

Actually, the base coordinate system $\mathscr{F}_{0}$ is fixed with respect to the earth. The outer axis of the turntable is aligned along the $y$-axis of the outer axis coordinate system $\mathscr{F}_{1}$. The inner axis of the turntable is aligned along the $z$ axis of the inner axis coordinate system $\mathscr{F}_{3}$. Since the GyroWheel is attached to the inner frame of the turntable, the GyroWheel coordinate system $\mathscr{F}_{4}$ is coincident with $\mathscr{F}_{3}$. The relations between these coordinate systems and the error components of the turntable system are expressed in Table 3. $\phi_{1}, \phi_{2}$ are the rotation angles about the outer axis and inner axis, respectively.

The relative position and orientation of one coordinate system with respect to another coordinate system can be modeled using a homogeneous transformation matrix (HTM), denoted as ${ }^{i} T_{j}, i, j=0,1,2,3,4$. The presuperscript represents the coordinate system we are transferring from, and the postsubscript represents the coordinate system we want the results to be represented in.

Hence, the transform relation between $\mathscr{F}_{1}$ and $\mathscr{F}_{0}$ can be calculated with the assumption of small angular errors:

$$
{ }^{0} \mathbf{T}_{1}=\left[\begin{array}{cccc}
\cos \phi_{1} & 0 & \sin \phi_{1} & 0 \\
0 & 1 & 0 & 0 \\
-\sin \phi_{1} & 0 & \cos \phi_{1} & 0 \\
0 & 0 & 0 & 1
\end{array}\right] \cdot\left[\begin{array}{cccc}
1 & -\Delta \alpha_{z 1} & \Delta \phi_{y 1} & 0 \\
\Delta \alpha_{z 1} & 1 & -\Delta \alpha_{x 1} & 0 \\
-\Delta \phi_{y 1} & \Delta \alpha_{x 1} & 1 & 0 \\
0 & 0 & 0 & 1
\end{array}\right] .
$$

The transform relation between $\mathscr{F}_{2}$ and $\mathscr{F}_{1}$ can be calculated as follows:

$$
{ }^{1} \mathbf{T}_{2}=\left[\begin{array}{cccc}
1 & 0 & \Delta \varepsilon_{y} & 0 \\
0 & 1 & -\Delta \varepsilon_{x} & 0 \\
-\Delta \varepsilon_{y} & \Delta \varepsilon_{x} & 1 & 0 \\
0 & 0 & 0 & 1
\end{array}\right] \cdot\left[\begin{array}{cccc}
1 & 0 & 0 & \Delta \eta \\
0 & 1 & 0 & 0 \\
0 & 0 & 1 & 0 \\
0 & 0 & 0 & 1
\end{array}\right]
$$




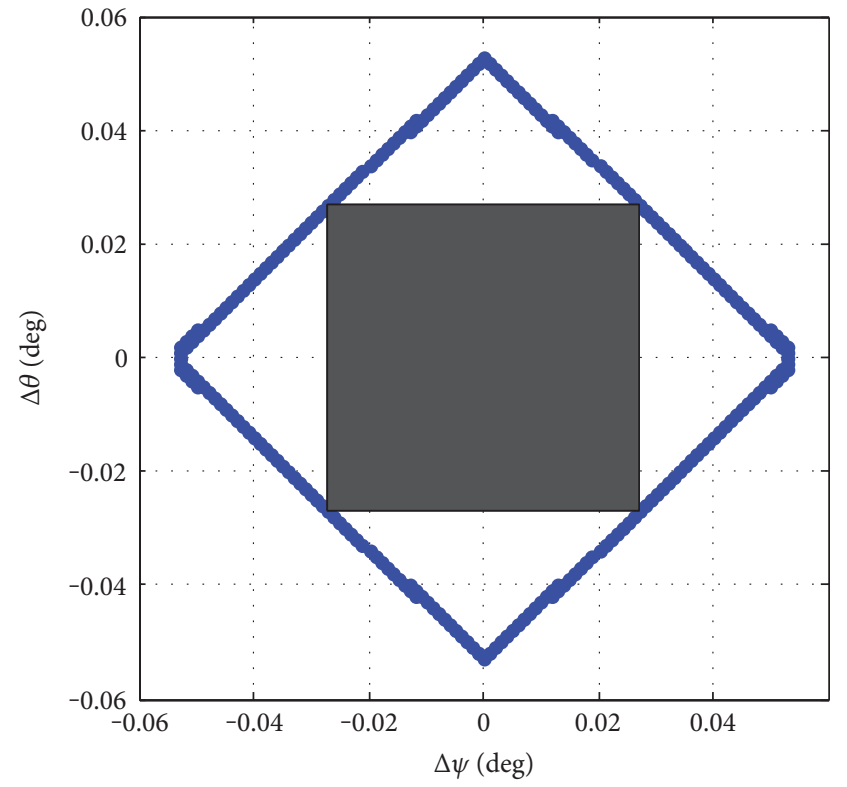

(a) $x$-axis

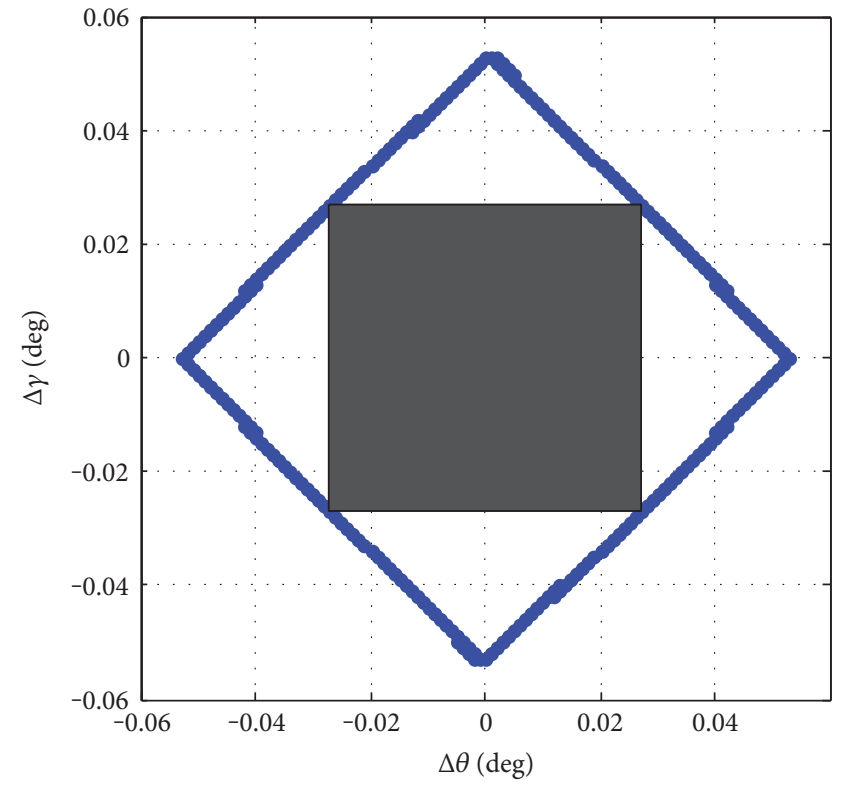

(b) $y$-axis

FIGURE 5: Lines of intersection.

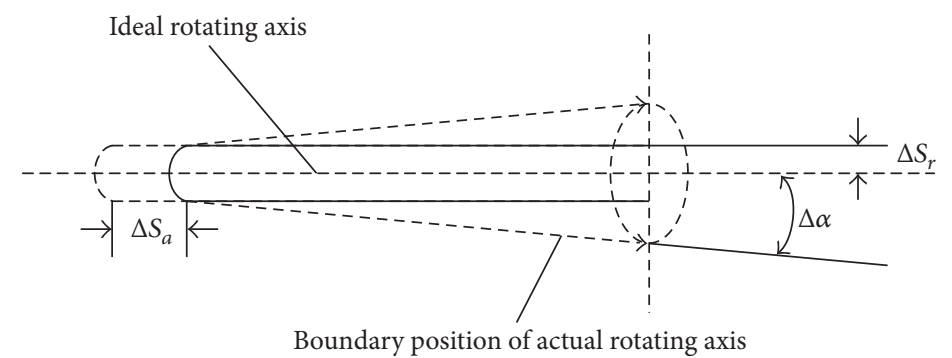

Figure 6: Schematic of a wobble error.

TABle 2: Error components of a two-axis turntable system.

\begin{tabular}{lcc}
\hline Location of error components & Symbols & Definitions \\
\hline \multirow{2}{*}{ Outer axis } & $\Delta \phi_{y 1}$ & Position error of outer axis \\
& $\Delta \alpha_{x 1}$ & Wobble error component of outer axis about $x$-axis \\
& $\Delta \alpha_{z 1}$ & Wobble error component of outer axis about $z$-axis \\
Inner axis & $\Delta \phi_{z 2}$ & Position error of inner axis \\
& $\Delta \alpha_{x 2}$ & Wobble error component of inner axis about $x$-axis \\
Between outer and inner axes & $\Delta \alpha_{y 2}$ & Wobble error component of inner axis about $y$-axis \\
& $\Delta \varepsilon_{x}$ & Orthogonality error component about $x$-axis \\
& $\Delta \varepsilon_{y}$ & Orthogonality error component about $y$-axis
\end{tabular}



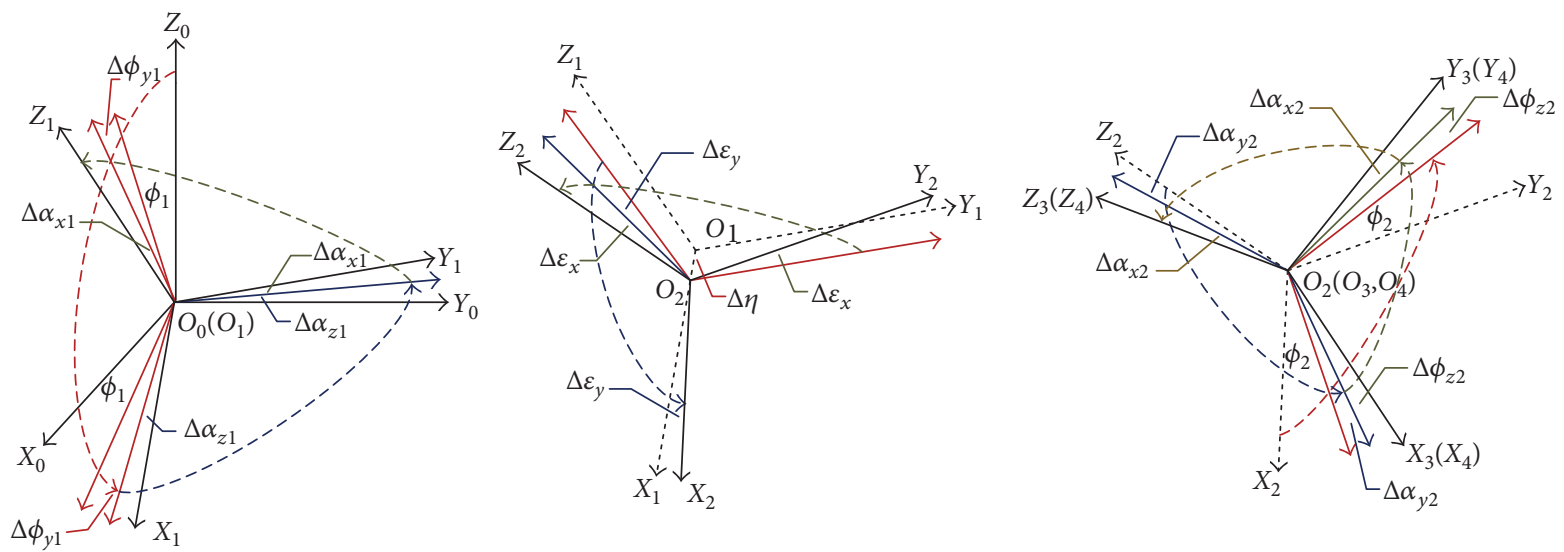

FIGURE 7: An illustration of the coordinate systems.

TABLE 3: Rotational and translational motion relations between adjacent coordinate systems.

\begin{tabular}{cc}
\hline$i$ & $\mathscr{F}_{i} \rightarrow \mathscr{F}_{i+1}$ \\
\hline 0 & A rotation $\phi_{1}$ about $y$-axis, followed by a rotation $\Delta \phi_{y 1}$ about $y$-axis \\
A rotation $\Delta \alpha_{z 1}$ about $z$-axis and then a rotation $\Delta \alpha_{x 1}$ about $x$-axis
\end{tabular}

And the transform relation between $\mathscr{F}_{3}$ and $\mathscr{F}_{2}$ is given below:

${ }^{2} \mathbf{T}_{3}=\left[\begin{array}{cccc}\cos \phi_{2} & -\sin \phi_{2} & 0 & 0 \\ \sin \phi_{2} & \cos \phi_{2} & 0 & 0 \\ 0_{1} & 0 & 1 & 0 \\ 0 & 0 & 0 & 1\end{array}\right] \cdot\left[\begin{array}{cccc}1 & -\Delta \phi_{z 2} & \Delta \alpha_{y 2} & 0 \\ \Delta \phi_{z 2} & 1 & -\Delta \alpha_{x 2} & 0 \\ -\Delta \alpha_{y 2} & \Delta \alpha_{x 2} & 1 & 0 \\ 0 & 0 & 0 & 1\end{array}\right]$.

As stated above, the transform relation between $\mathscr{F}_{4}$ and $\mathscr{F}_{3}$ is written in the following form:

$$
{ }^{3} \mathbf{T}_{4}=\mathbf{I}_{4 \times 4}
$$

We introduce homogeneous transformation matrixes $\mathbf{T}_{d}, \mathbf{T}_{t}$ to denote the ideal and actual transform relations between the GyroWheel and the base coordinate system. $\mathbf{T}_{d}$ is obtained through a series of perfect rotations $\left(\phi_{1}, \phi_{2}\right)$ and is given by the following:

$$
\mathbf{T}_{d}=\left[\begin{array}{cccc}
\cos \phi_{1} & 0 & \sin \phi_{1} & 0 \\
0 & 1 & 0 & 0 \\
-\sin \phi_{1} & 0 & \cos \phi_{1} & 0 \\
0 & 0 & 0 & 1
\end{array}\right] \cdot\left[\begin{array}{cccc}
\cos \phi_{2} & -\sin \phi_{2} & 0 & 0 \\
\sin \phi_{2} & \cos \phi_{2} & 0 & 0 \\
0 & 0 & 1 & 0 \\
0 & 0 & 0 & 1
\end{array}\right] .
$$

In presence of the turntable errors, $\mathbf{T}_{t}$ can be calculated by matrix multiplication:

$$
\mathbf{T}_{t}=\prod_{i=0}^{3}{ }^{i} \mathbf{T}_{i+1}
$$

The top left corner $3 \times 3$ matrixes of $\mathbf{T}_{d}, \mathbf{T}_{t}$ denoted by $\mathbf{R}_{d}, \mathbf{R}_{t}$ represent the ideal and actual direction cosine matrixes. Since $\mathbf{R}_{d}, \mathbf{R}_{t}$ are orthogonal matrixes, the orientation error of the GyroWheel caused by the turntable errors can be expressed as follows:

$$
\Delta \mathbf{R}=\mathbf{R}_{d}^{T} \mathbf{R}_{t} .
$$

According to (23) and (7), we have the following:

$$
\begin{aligned}
& \Delta \psi=\Delta R_{13}, \\
& \Delta \theta=\Delta R_{21}, \\
& \Delta \gamma=\Delta R_{32},
\end{aligned}
$$

where $\Delta R_{i j}$ represents the element in the $i$ th row and $j$ th column of matrix $\Delta \mathbf{R}$. Substituting (15)-(21) into (22) yields the following expression:

$$
\begin{aligned}
& \Delta \psi=f_{\psi}(\phi, \chi), \\
& \Delta \theta=f_{\theta}(\phi, \chi), \\
& \Delta \gamma=f_{\gamma}(\phi, \chi),
\end{aligned}
$$


where $f_{\psi}, f_{\theta}, f_{\gamma}$ are nonlinear functions of the rotation angles and the turntable error components, $\phi=\left[\phi_{1} \phi_{2}\right]^{T}$ represents the rotation angle vector, and $\chi=\left[\begin{array}{llll}\Delta \phi_{y 1} & \Delta \phi_{z 2} & \Delta \alpha_{x 1} & \Delta \alpha_{z 1}\end{array}\right.$ $\left.\Delta \alpha_{x 2} \Delta \alpha_{y 2} \Delta \varepsilon_{x} \Delta \varepsilon_{y}\right]^{T}$ represents the turntable error vector. According to (25), the intersection error has no effect on the orientation error of the GyroWheel.

To simplify the analysis, (25) is rewritten into the following form with small angle approximation of the errors:

$$
\begin{aligned}
\Delta \psi= & f_{\psi}\left(\phi_{2}, \chi_{\psi}\right)=\Delta \phi_{y 1} \cos \phi_{2}-\Delta \alpha_{x 1} \sin \phi_{2}+\Delta \alpha_{y 2} \\
& -\Delta \varepsilon_{x} \sin \phi_{2}+\Delta \varepsilon_{y} \cos \phi_{2}, \\
\Delta \theta= & f_{\theta}\left(\chi_{\theta}\right)=\Delta \phi_{z 2}+\Delta \alpha_{z 1}, \\
\Delta \gamma= & f_{\gamma}\left(\phi_{2}, \chi_{\gamma}\right)=\Delta \phi_{y 1} \sin \phi_{2}+\Delta \alpha_{x 1} \cos \phi_{2}+\Delta \alpha_{x 2} \\
& +\Delta \varepsilon_{x} \cos \phi_{2}+\Delta \varepsilon_{y} \sin \phi_{2},
\end{aligned}
$$

where

$$
\begin{aligned}
& \chi_{\gamma}=\left[\begin{array}{lllll}
\Delta \phi_{y 1} & \Delta \alpha_{x 1} & \Delta \alpha_{x 2} & \Delta \varepsilon_{x} & \Delta \varepsilon_{y}
\end{array}\right]^{T}, \\
& \chi_{\theta}=\left[\begin{array}{lllll}
\Delta \phi_{z 2} & \Delta \alpha_{z 1}
\end{array}\right]^{T}, \\
& \chi_{\psi}=\left[\begin{array}{lllll}
\Delta \phi_{y 1} & \Delta \alpha_{x 1} & \Delta \alpha_{y 2} & \Delta \varepsilon_{x} & \Delta \varepsilon_{y}
\end{array}\right]^{T} .
\end{aligned}
$$

\section{Error Allocation of the Turntable System}

4.1. Sensitivity Analysis and Constraints Analysis. To ensure the orientation accuracy and perform error allocation of the turntable system, sensitivities of the orientation error to the individual errors and the manufacturing capability should be considered.

According to (23), sensitivities of the orientation error can be analyzed:

$$
\begin{aligned}
C_{\psi i} & =\left|\frac{\partial f_{\psi}}{\partial \chi_{i}}\right|, \\
C_{\theta i} & =\left|\frac{\partial f_{\theta}}{\partial \chi_{i}}\right|, \\
C_{\gamma i} & =\left|\frac{\partial f_{\gamma}}{\partial \chi_{i}}\right|, \\
i & =1,2, \ldots, 8 .
\end{aligned}
$$

$\chi_{i}$ represents the $i$ th element of the turntable error vector $\chi$, and $C_{\psi i}, C_{\theta i}, C_{\gamma i}$ represent the sensitivities of the orientation error to the individual error components. Given that $C_{\psi i}$, $C_{\theta i}, C_{\gamma i}$ vary with the rotation angles, we here consider the average values, which are given in Figure 8 . The values represent how sensitive the orientation error is to the individual error components. For example, if the orientation error is more sensitive to error component $\chi_{i}$ than to error component $\chi_{j}, \chi_{i}$ is required to be smaller than $\chi_{j}$ to achieve a reasonable error allocation.
Additionally, with the limitation of technological level and manufacturing costs in practical engineering, the manufacturing capability determines the maximum accuracy that the turntable can reach. Therefore, it gives the lower bounds of the error components of the turntable system, namely, the error components should satisfy the constraints:

$$
\left|\chi_{i}\right| \geq \mathrm{LB}_{i}, \quad i=1,2, \ldots, 8
$$

$\mathrm{LB}_{i}$ are the lower bounds of the error components.

4.2. Error Allocation Method for Turntable Systems. For the error allocation problem of the turntable system, we expect to find the upper bounds $\mathrm{UB}_{i}, i=1,2, \cdots, 8$. The turntable system should reach the required orientation accuracy at any rotation angles when the error components satisfy the constraints, namely,

$$
\begin{aligned}
& \left|f_{\gamma}(\phi, \chi)\right| \leq I_{\gamma}, \\
& \left|f_{\theta}(\phi, \chi)\right| \leq I_{\theta}, \\
& \left|f_{\psi}(\phi, \chi)\right| \leq I_{\psi}, \\
& \forall \phi \in \Omega_{\phi}, \forall \chi \in \Omega_{\chi},
\end{aligned}
$$

where $\Omega_{\phi}=\left\{\phi \mid 0 \leq \phi_{i} \leq 2 \pi, i=1,2\right\}$ and $\Omega_{\chi}=\left\{\chi\left|\mathrm{LB}_{i} \leq\right| \chi_{i} \mid\right.$ $\left.\leq \mathrm{UB}_{i}, i=1,2, \ldots, 8\right\}$.

With the simplification of the orientation error model given by (26), the error allocation of the turntable system can be described by the following optimization problems:

$$
\begin{array}{ll}
\min _{\chi_{\gamma}} & J_{\gamma}=\sum_{i=1}^{5} W_{\gamma i}^{2} \frac{1}{\chi_{\gamma i}^{2}}, \\
\text { s.t. } & f_{\gamma}\left(\phi_{2}, \chi_{\gamma}\right) \leq I_{\gamma}, \quad \forall \phi_{2} \in\left[0, \frac{\pi}{2}\right], \\
& \chi_{\gamma i} \geq \mathrm{LB}_{\gamma i}, \quad i=1,2, \ldots, 5, \\
\min _{\chi_{\theta}} & J_{\theta}=\sum_{i=1}^{2} W_{\theta i}^{2} \frac{1}{\chi_{\theta i}^{2}}, \\
\text { s.t. } & f_{\theta}\left(\chi_{\theta}\right) \leq I_{\theta}, \\
& \chi_{\theta i} \geq \mathrm{LB}_{\theta i}, \quad i=1,2, \\
\min _{\chi_{\psi}} & J_{\psi}=\sum_{i=1}^{5} W_{\psi i}^{2} \frac{1}{\chi_{\psi i}^{2}}, \\
\text { s.t. } & f_{\psi}\left(\phi_{2}, \chi_{\psi}\right) \leq I_{\psi}, \quad \forall \phi_{2} \in\left[\frac{3 \pi}{2}, 2 \pi\right], \\
& \chi_{\psi i} \geq \mathrm{LB}_{\psi i}, \quad i=1,2, \ldots, 5,
\end{array}
$$

where $\chi_{\gamma i}, \chi_{\theta i}, \chi_{\psi i}$ are the ith elements of the vectors $\chi_{\gamma}$, $\chi_{\theta}, \chi_{\psi}$, respectively. $W_{\gamma}, W_{\theta}, W_{\psi}$ are weighted coefficients; the values of which are the multiplicative inverse of the corresponding sensitivities given in Figure 8 . By solving the optimization problems shown in (31)-(35), the optimal 


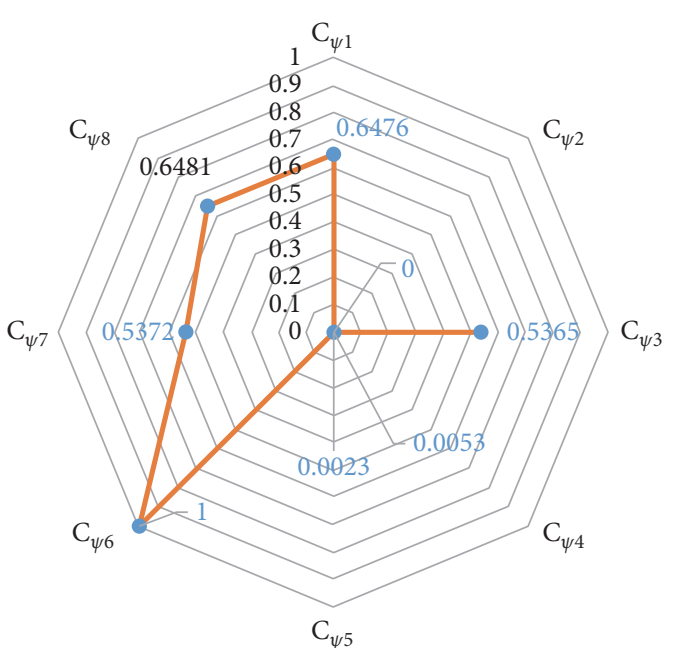

(a) $\Delta \psi$

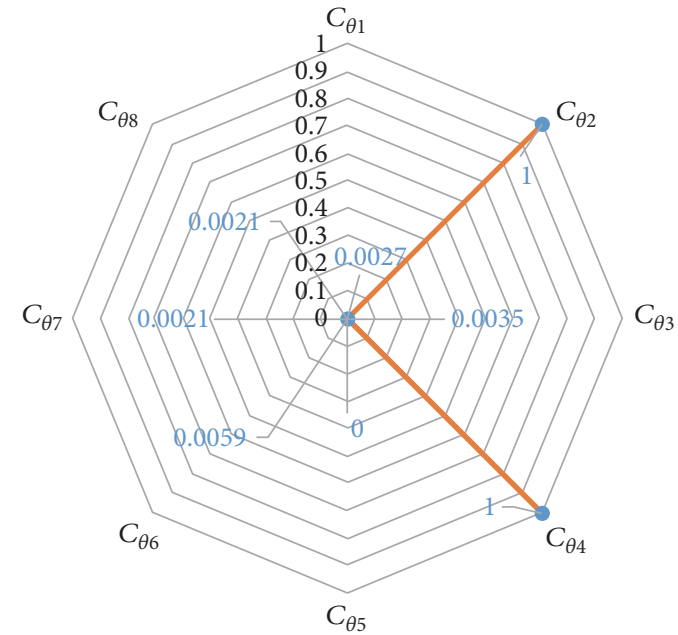

(b) $\Delta \theta$

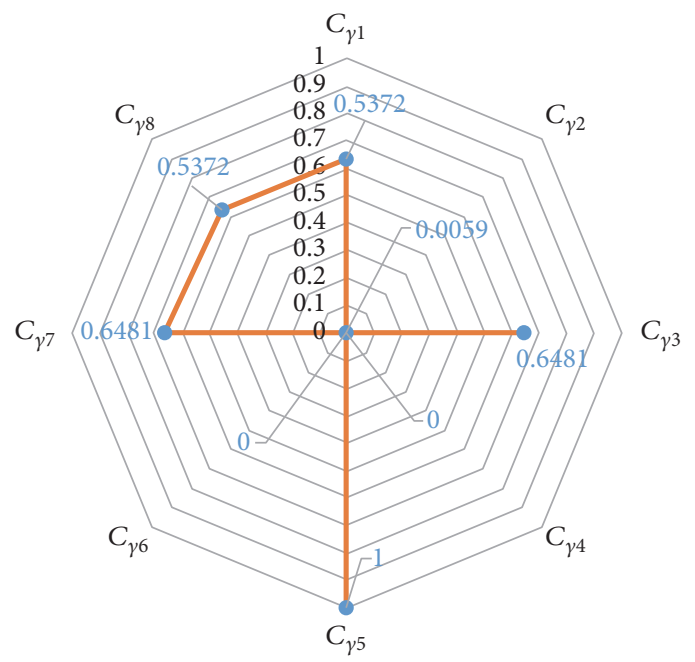

(c) $\Delta \gamma$

FIgURE 8: Sensitivities of orientation error to individual error components.

solutions $\chi_{\gamma}^{*}, \chi_{\theta}^{*}, \chi_{\psi}^{*}$ are obtained respectively. Then, the upper bounds of the turntable errors can be given by

$$
\begin{gathered}
\mathrm{UB}_{1}=\min \left(\chi_{\gamma 1}^{*}, \chi_{\psi 1}^{*}\right), \\
\mathrm{UB}_{2}=\chi_{\theta 1}^{*}, \\
\mathrm{UB}_{3}=\min \left(\chi_{\gamma 2}^{*}, \chi_{\psi 2}^{*}\right), \\
\mathrm{UB}_{4}=\chi_{\theta 2}^{*}, \\
\mathrm{UB}_{5}=\chi_{\gamma 3}^{*}, \\
\mathrm{UB}_{6}=\chi_{\psi 3}^{*}, \\
\mathrm{UB}_{7}=\min \left(\chi_{\gamma 4}^{*}, \chi_{\psi 4}^{*}\right), \\
\mathrm{UB}_{8}=\min \left(\chi_{\gamma 5}^{*}, \chi_{\psi 5}^{*}\right) .
\end{gathered}
$$

According to (31)-(36), the error allocation of the turntable system can be accomplished. The error allocation results $\mathrm{UB}_{i}, i=1,2, \ldots, 8$, are proven below.
Lemma 1. Consider a turntable system with the orientation error model shown in (26). The error allocation results $\mathrm{UB}_{i}$, $i=1,2, \ldots, 8$, are obtained from (31)-(36). $\forall \phi \in \Omega_{\phi}, \forall \chi \in$ $\Omega_{\chi}$, where $\Omega_{\phi}=\left\{\phi \mid 0 \leq \phi_{i} \leq 2 \pi i=12\right\}$ and $\Omega_{\chi}=\left\{\chi \mid \mathrm{LB}_{i} \leq\right.$ $\left.\left|\chi_{i}\right| \leq \mathrm{UB}_{i} i=12 \cdots 8\right\}$, it is always true that $\left|f_{\gamma}(\phi, \chi)\right| \leq$ $I_{\gamma},\left|f_{\theta}(\phi, \chi)\right| \leq I_{\theta},\left|f_{\psi}(\phi, \chi)\right| \leq I_{\psi}$.

Proof

Case 1. $\forall \phi_{2} \in[0,(\pi / 2)]$, then

$$
\begin{aligned}
\left|f_{\gamma}(\phi, \chi)\right|= & \mid \Delta \phi_{y 1} \sin \phi_{2}+\Delta \alpha_{x 1} \cos \phi_{2}+\Delta \alpha_{x 2}+\Delta \varepsilon_{x} \cos \phi_{2} \\
& +\Delta \varepsilon_{y} \sin \phi_{2}|\leq| \Delta \phi_{y 1}\left|\sin \phi_{2}+\right| \Delta \alpha_{x 1} \mid \cos \phi_{2} \\
& +\left|\Delta \alpha_{x 2}\right|+\left|\Delta \varepsilon_{x}\right| \cos \phi_{2}+\left|\Delta \varepsilon_{y}\right| \sin \phi_{2} \leq \chi_{\gamma 1}^{*} \sin \phi_{2} \\
& +\chi_{\gamma 2}^{*} \cos \phi_{2}+\chi_{\gamma 3}^{*}+\chi_{\gamma 4}^{*} \cos \phi_{2}+\chi_{\gamma 5}^{*} \sin \phi_{2} \\
= & f_{\gamma}\left(\phi_{2}, \chi_{\gamma}^{*}\right) \leq I_{\gamma} .
\end{aligned}
$$


Case 2. $\forall \phi_{2} \in[(\pi / 2), \pi]$, denote $\widehat{\phi}_{2}=\pi-\phi_{2}$; then $\forall \widehat{\phi}_{2} \epsilon$ $[0,(\pi / 2)]$,

$$
\begin{aligned}
\left|f_{\gamma}(\phi, \chi)\right|= & \mid \Delta \phi_{y 1} \sin \phi_{2}+\Delta \alpha_{x 1} \cos \phi_{2}+\Delta \alpha_{x 2}+\Delta \varepsilon_{x} \cos \phi_{2} \\
& +\Delta \varepsilon_{y} \sin \phi_{2}|\leq| \Delta \phi_{y 1}\left|\sin \widehat{\phi}_{2}+\right| \Delta \alpha_{x 1} \mid \cos \widehat{\phi}_{2} \\
& +\left|\Delta \alpha_{x 2}\right|+\left|\Delta \varepsilon_{x}\right| \cos \widehat{\phi}_{2}+\left|\Delta \varepsilon_{y}\right| \sin \widehat{\phi}_{2} \leq \chi_{\gamma 1}^{*} \sin \widehat{\phi}_{2} \\
& +\chi_{\gamma 2}^{*} \cos \widehat{\phi}_{2}+\chi_{\gamma 3}^{*}+\chi_{\gamma 4}^{*} \cos \widehat{\phi}_{2}+\chi_{\gamma 5}^{*} \sin \widehat{\phi}_{2} \\
= & f_{\gamma}\left(\widehat{\phi}_{2}, \chi_{\gamma}^{*}\right) \leq I_{\gamma} .
\end{aligned}
$$

Case 3. $\forall \phi_{2} \in[\pi,((3 \pi) / 2)]$, denote $\widehat{\phi}_{2}=\phi_{2}-\pi$; then $\forall \widehat{\phi}_{2} \in[0,(\pi / 2)]$,

$$
\begin{aligned}
\left|f_{\gamma}(\phi, \chi)\right|= & \mid \Delta \phi_{y 1} \sin \phi_{2}+\Delta \alpha_{x 1} \cos \phi_{2}+\Delta \alpha_{x 2}+\Delta \varepsilon_{x} \cos \phi_{2} \\
& +\Delta \varepsilon_{y} \sin \phi_{2}|\leq| \Delta \phi_{y 1}\left|\sin \widehat{\phi}_{2}+\right| \Delta \alpha_{x 1} \mid \cos \widehat{\phi}_{2} \\
& +\left|\Delta \alpha_{x 2}\right|+\left|\Delta \varepsilon_{x}\right| \cos \widehat{\phi}_{2}+\left|\Delta \varepsilon_{y}\right| \sin \widehat{\phi}_{2} \leq \chi_{\gamma 1}^{*} \sin \widehat{\phi}_{2} \\
& +\chi_{\gamma 2}^{*} \cos \widehat{\phi}_{2}+\chi_{\gamma 3}^{*}+\chi_{\gamma 4}^{*} \cos \widehat{\phi}_{2}+\chi_{\gamma 5}^{*} \sin \widehat{\phi}_{2} \\
= & f_{\gamma}\left(\widehat{\phi}_{2}, \chi_{\gamma}^{*}\right) \leq I_{\gamma} .
\end{aligned}
$$

Case 4. $\forall \phi_{2} \in[((3 \pi) / 2), 2 \pi]$, denote $\widehat{\phi}_{2}=2 \pi-\phi_{2}$; then $\forall \widehat{\phi}_{2} \in[0,(\pi / 2)]$,

$$
\begin{aligned}
&\left|f_{\gamma}(\phi, \chi)\right|= \mid \Delta \phi_{y 1} \sin \phi_{2}+\Delta \alpha_{x 1} \cos \phi_{2}+\Delta \alpha_{x 2}+\Delta \varepsilon_{x} \cos \phi_{2} \\
&+\Delta \varepsilon_{y} \sin \phi_{2}|\leq| \Delta \phi_{y 1}\left|\sin \widehat{\phi}_{2}+\right| \Delta \alpha_{x 1} \mid \cos \widehat{\phi}_{2} \\
&+\left|\Delta \alpha_{x 2}\right|+\left|\Delta \varepsilon_{x}\right| \cos \widehat{\phi}_{2}+\left|\Delta \varepsilon_{y}\right| \sin \widehat{\phi}_{2} \leq \chi_{\gamma 1}^{*} \sin \widehat{\phi}_{2} \\
&+\chi_{\gamma 2}^{*} \cos \widehat{\phi}_{2}+\chi_{\gamma 3}^{*}+\chi_{\gamma 4}^{*} \cos \widehat{\phi}_{2}+\chi_{\gamma 5}^{*} \sin \widehat{\phi}_{2} \\
&= f \\
& \gamma\left(\widehat{\phi}_{2}, \chi_{\gamma}^{*}\right) \leq I_{\gamma} .
\end{aligned}
$$

In summary, $\forall \phi \in \Omega_{\phi}, \forall \chi \in \Omega_{\chi}$, we have $\left|f_{\gamma}(\phi, \chi)\right| \leq I_{\gamma}$, where $\Omega_{\phi}=\left\{\phi \mid 0 \leq \phi_{i} \leq 2 \pi, i=1,2\right\}$ and $\Omega_{\chi}=\left\{\chi\left|\mathrm{LB}_{i} \leq\right| \chi_{i} \mid\right.$ $\left.\leq \mathrm{UB}_{i}, i=1,2, \ldots, 8\right\}$.

Similarly, $\left|f_{\theta}(\phi, \chi)\right| \leq I_{\theta},\left|f_{\psi}(\phi, \chi)\right| \leq I_{\psi}$ can be demonstrated as well.

4.3. Example. Take the GyroWheel with the required calibration accuracy of $0.01^{\circ} / \mathrm{h}$ as an example. According to the analysis in Section 2, the requirement of the orientation accuracy is given in (16). Using the error allocation method proposed in this section, as shown in (31)-(36), the allocation results are given in Table 4.
TABLE 4: Error allocation results of the turntable system.

\begin{tabular}{lcc}
\hline Error sources & Symbols & $\begin{array}{c}\text { Accuracy requirements } \\
\text { (arc minute) }\end{array}$ \\
\hline Position error & $\Delta \phi_{y 1}$ & 0.4235 \\
& $\Delta \phi_{z 2}$ & 0.7800 \\
\hline \multirow{2}{*}{ Wobble error } & $\Delta \alpha_{x 1}$ & 0.4234 \\
& $\Delta \alpha_{z 1}$ & 0.7800 \\
& $\Delta \alpha_{x 2}$ & 0.2825 \\
Orthogonality error & $\Delta \alpha_{y 2}$ & 0.2824 \\
\hline
\end{tabular}

As seen in Table 4, the $x$-axis wobble error component of the inner axis $\Delta \alpha_{x 2}$ and the $y$-axis wobble error component of the inner axis $\Delta \alpha_{y_{2}}$ have higher accuracy requirements than the other error components. This is due to the fact that the orientation error is more sensitive to $\Delta \alpha_{x 2}$ and $\Delta \alpha_{y 2}$, as shown in Figure 8. According to Figure 8, only $\Delta \theta$ is sensitive to the position error of the inner axis $\Delta \phi_{z 2}$ and the $z$-axis wobble error component of the outer axis $\Delta \alpha_{z 1}$, and $\Delta \psi$ and $\Delta \gamma$ are insensitive to $\Delta \phi_{z 2}$ and $\Delta \alpha_{z 1}$. Meanwhile, $\Delta \theta$ is affected only by $\Delta \phi_{z 2}$ and $\Delta \alpha_{z 1}$; thus, $\Delta \phi_{z 2}$ and $\Delta \alpha_{z 1}$ have lower accuracy requirements. The error allocation results provide a basis for the selection of the suitable turntable and are of great significance to guide the calibration tests and improve the calibration accuracy of the GyroWheel.

Given that the error allocation of the turntable system is accomplished based on certain simplifications, Monte Carlo simulations are performed to verify the effectiveness of the error allocation results for the original orientation error model as shown in (25). According to the allocation results given in Table 4 , values of the error components satisfying the accuracy requirements $\mathrm{LB}_{i} \leq\left|\chi_{i}\right| \leq \mathrm{UB}_{i}, i=1,2, \ldots, 8$ are generated randomly by utilizing MATLAB. 10,000 times of numerical simulations are performed, and the orientation error is calculated for each simulation test. The distribution of $\Delta \psi, \Delta \theta, \Delta \gamma$ describing the orientation error for the 10,000 times of simulations is shown in Figure 9.

It is seen in Figure 9 that the angular errors $\Delta \psi, \Delta \theta$, $\Delta \gamma$ always satisfy the corresponding accuracy requirements in the repeated random simulation tests, which verifies the validity of the proposed error allocation method in this section.

\section{Conclusions}

Error analysis and error allocation problems in calibration tests are studied for GyroWheel in this paper. The relationships between the rate-sensing errors of GyroWheel and the orientation error are obtained, and the orientation error induced by the turntable errors is modelled based on rigid 


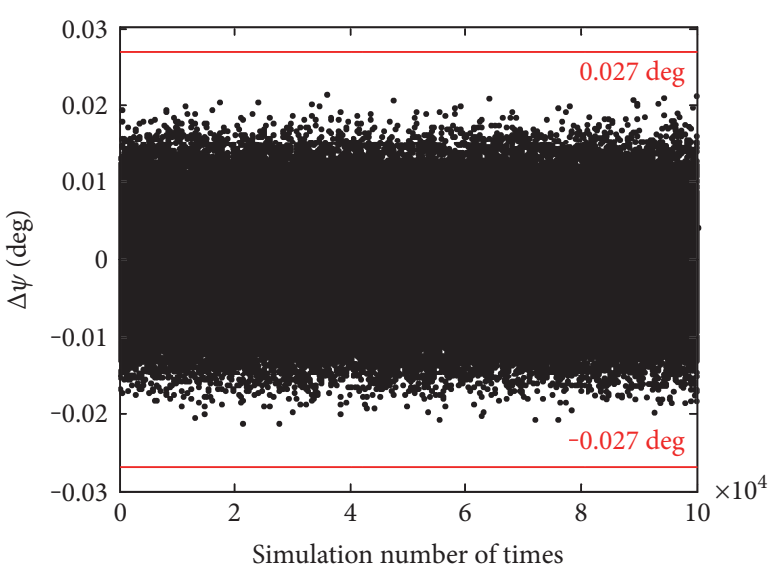

(a) $\Delta \psi$

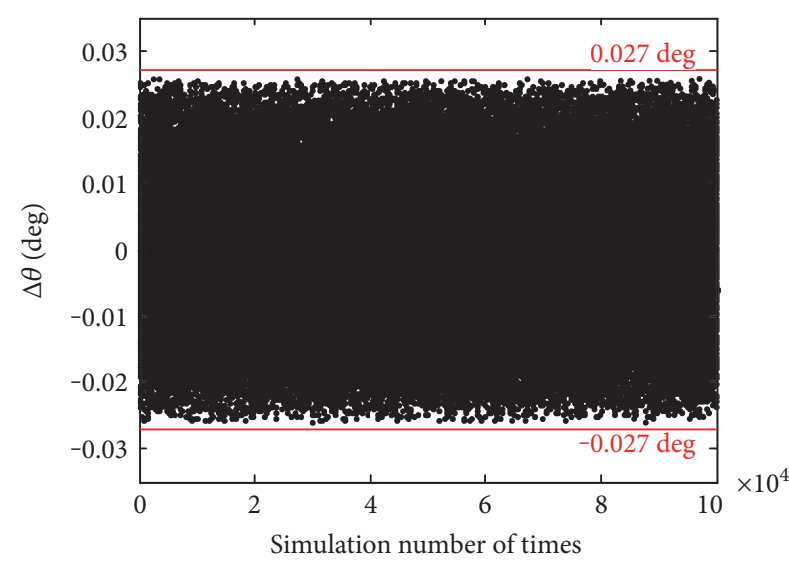

(b) $\Delta \theta$

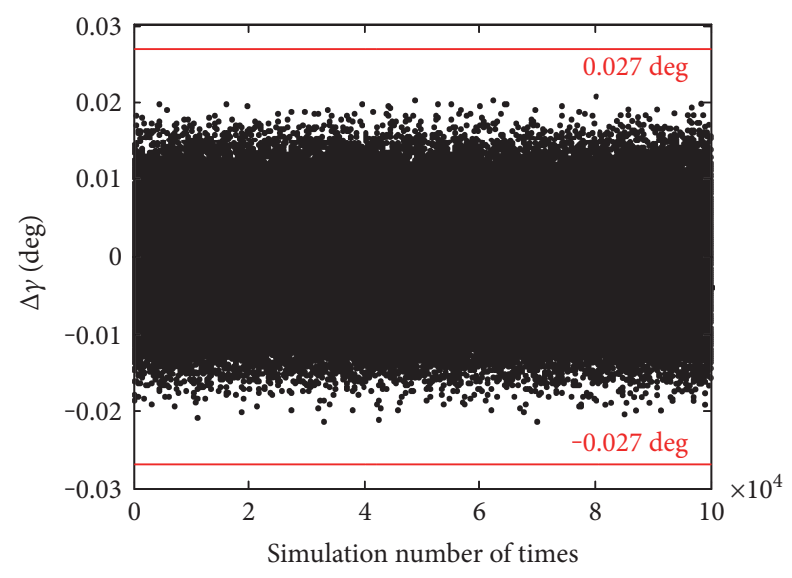

(c) $\Delta \gamma$

Figure 9: Distribution of orientation error.

body kinematics. A practical error allocation method is developed to determine the accuracy requirement of the test turntable. The salient features and contributions of this work are as follows:

(1) The error analysis for the GyroWheel calibration tests and the turntable system provides a way to express the effects of various turntable errors on the calibration accuracy quantitatively. Of all the error components, the wobble error of the inner axis contributes most to the calibration error.

(2) The proposed error allocation method provides a theoretical basis for the selection of the suitable turntable used in the calibration tests. With the considerations of the sensitivities of the orientation error to the individual error components, the requirements of these error components are determined according to the different sensitivities. Compared with the traditional allocation method assuming the equivalent effects of the errors, the proposed method is more reasonable and is beneficial to reducing the costs of calibration tests.
Although the study is focused on a two-axis turntable for GyroWheel calibration, it can be easily applied to other inertial instruments and other types of turntables, and it holds true for the cases of other calibration equipment such as centrifuges as well.

To further enhance the calibration accuracy of the GyroWheel, future research will focus on the compensation of the turntable errors.

\section{Conflicts of Interest}

The authors declare that there is no conflict of interests regarding the publication of this paper.

\section{Acknowledgments}

The research presented in this document is supported by the National Natural Science Foundation of China (NNSF) under Grant nos. 61427809 and 61773138, the China Postdoctoral Science Foundation under Grant 2015M571415, and the Heilongjiang Postdoctoral Foundation under Grant LBH-Z14088. 


\section{References}

[1] W. A. Bezouska, M. R. Aherne, J. T. Barrett, and S. J. Schultz, Demonstration of Technologies for Autonomous MicroSatellite Assembly, AIAA SPACE 2009 Conference \& Exposition, Pasadena, California, 2009.

[2] C. Balty and J. D. Gayrard, Flexible Satellites: A New Challenge for the Communication Satellite Industry, AIAA International Communications Satellite Systems Conference, 25th edition, 2007.

[3] G. Tyc, D. A. Staley, W. R. Whitehead et al., GyroWheel ${ }^{\mathrm{TM}_{-} a n}$ Innovative New Actuator/Sensor for 3-Axis Spacecraft Attitude Control, Annual AIAA/USU Conference on Small Satellites, 13th edition, 1999.

[4] T. H. Paul, Development and Testing of a GyroWheel Based Control System for the SCISAT-1 Scientific Satellite, Carleton University, Canada, Master Dissertation, 2003.

[5] J. M. Hall, Calibration of an Innovative Rate Sensing /Momentum Management Instrument for de-Tuned Operation and Temperature Effects, Carleton University, Canada, Master Dissertation, 2008.

[6] J. C. Ower, Analysis and Control System Design of an Innovative Tuned-Rotor Instrument, Carleton University, Canada, Doctor Dissertation, 2000.

[7] D. H. Titterton and J. L. Weston, Strapdown Inertial Navigation Technology, Institution of Electrical Engineers, 2nd edition, 2004.

[8] L. Fu, X. Yang, and L. L. Wang, "A novel calibration procedure for dynamically tuned gyroscope designed by D-optimal approach," Measurement, vol. 46, no. 9, pp. 31733180, 2013.

[9] Z. F. Syed, P. Aggarwal, C. Goodall, X. Niu, and N. El-Sheimy, "A new multi-position calibration method for MEMS inertial navigation systems," Measurement Science and Technology, vol. 18, no. 7, p. 1897, 2007.

[10] Y. Y. Zhao, H. Zhao, and X. Huo, "A new multi-position calibration method for the Gyrowheel using multiple objective particle swarm optimization algorithm," AIAA Guidance, Navigation, and Control Conference, pp. 1906-1916, 2017.

[11] L. A. DeMore, R. A. Peterson, L. B. Conley, H. Havliscek, and N. P. Andrianos, "Design study for a high-accuracy three-axis test table," Journal of Guidance, Control, and Dynamics, vol. 10, no. 1, pp. 104-114, 1987.

[12] A. C. Okafor and Y. M. Ertekin, "Derivation of machine tool error models and error compensation procedure for three axes vertical machining center using rigid body kinematics," International Journal of Machine Tools and Manufacture, vol. 40, no. 8, pp. 1199-1213, 2000.

[13] Y. Yao and Z. Y. Qu, "Derivation of generic error models procedure for 6-DOF motion system using multi-body kinematics," in 2006 1st International Symposium on Systems and Control in Aerospace and Astronautics, pp. 1267-1272, Harbin, China, January 2006.

[14] Z. Y. Qu and Y. Yao, "Derivation of error models and error compensation procedure for simulation turntable using multi-body kinematics," in IEEE International Conference Mechatronics and Automation, pp. 1408-1411, Niagara Falls, Ont., Canada, August 2005.

[15] S. M. Wang and S. Q. Ren, "Calibration of cross quadratic term of gyro accelerometer on centrifuge and error analysis," Aerospace Science and Technology, vol. 43, pp. 30-36, 2015.
[16] S. M. Wang and S. Q. Ren, "Relationship between calibration accuracy of error model coefficients of accelerometer and errors of precision centrifuge," Journal of Astronautics, vol. 33, no. 4, pp. 520-526, 2012.

[17] H. L. Zhang, Y. X. Wu, J. X. Lian, and W. Q. Wu, "Improved calibration scheme for high precision IMUs based on turntable error analysis," Journal of Chinese Inertial Technology, vol. 18, no. 1, pp. 129-134, 2010.

[18] Y. B. Li and M. Zeng, "Influence of three-axis turntable error source on pendulous integrating gyro accelerometer testing," Aviation Precision Manufacturing Technology, vol. 44, no. 2, pp. 28-31, 2008.

[19] G. P. Xiao, H. L. Zhang, M. Lv, W. Q. Wu, and Y. X. Wu, "Effect of turntable's orthogonal error and horizontal error on gyro calibration accuracy," Journal of System Simulation, vol. 20, pp. 370-373, 2009.

[20] Y. J. Zhao, X. H. Zhao, and W. M. Ge, "An algorithm for the accuracy synthesis of a 6-SPS parallel manipulator," Mechanical Science and Technology, vol. 23, no. 4, pp. 392-395, 2004.

[21] F. W. Pan, Z. S. Duan, L. L. He, and P. Wang, "Precision synthesis on novel 6DOF parallel robot based on fuzzy genetic algorithm," Journal of Machine Design, vol. 23, no. 9, pp. 4548, 2006.

[22] W. Wang and C. Yun, "Orthogonal experimental design to synthesize the accuracy of robotic mechanism," Journal of Mechanical Engineering, vol. 45, no. 11, pp. 18-24, 2009.

[23] W. Li, S. Q. Ren, and H. B. Zhao, "Influence of three-axis turntable error on gyro calibration accuracy," Electric Machines and Control, vol. 15, no. 10, pp. 101-106, 2011.

[24] S. Q. Ren and J. Z. Wang, "Data processing method of calculating wobble error with level instrument," Journal of Harbin Institute of Technology, vol. 38, no. 6, pp. 837-847, 2006. 


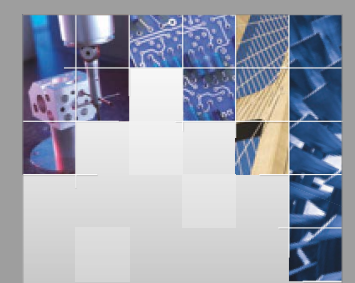

\section{Enfincering}
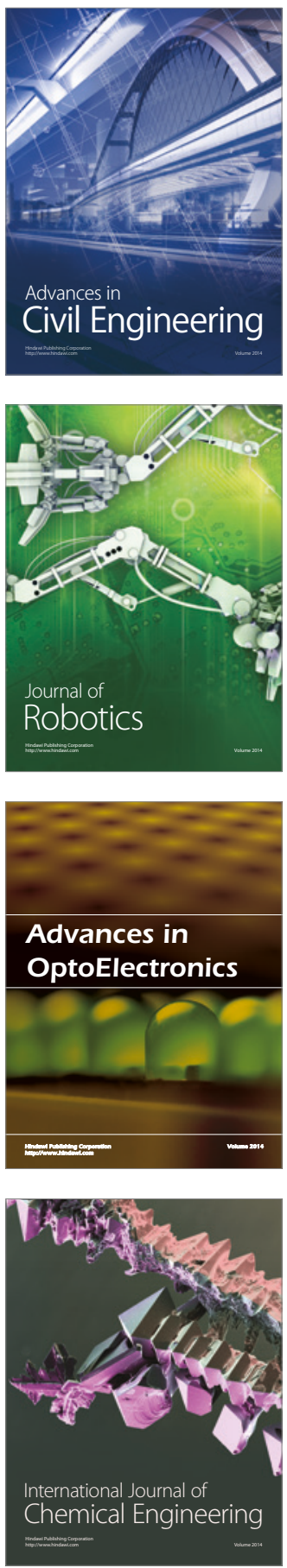

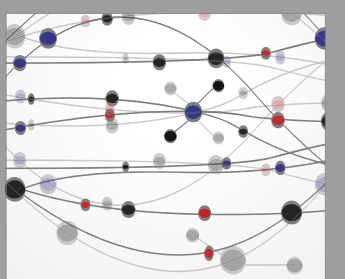

The Scientific World Journal

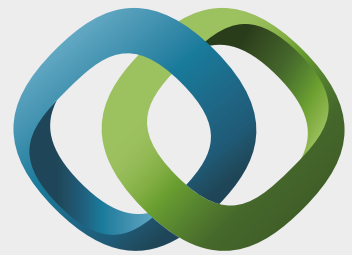

\section{Hindawi}

Submit your manuscripts at

https://www.hindawi.com
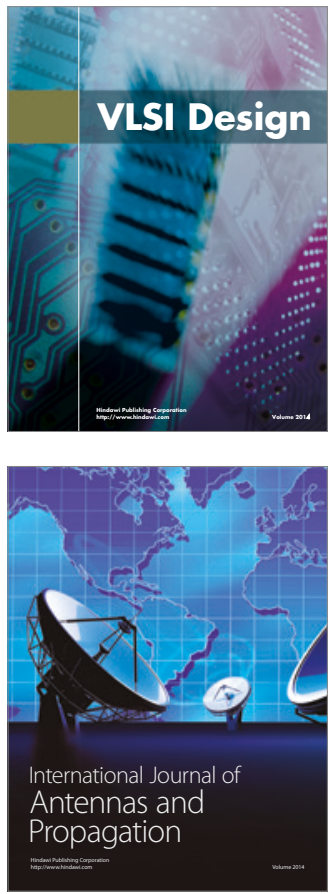

\section{Rotating}

Machinery
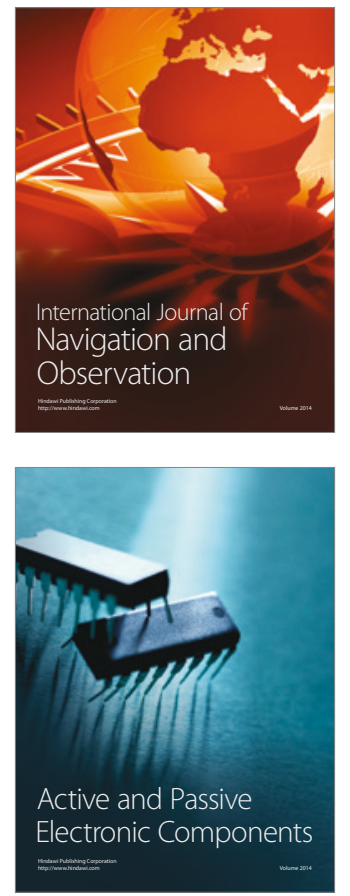
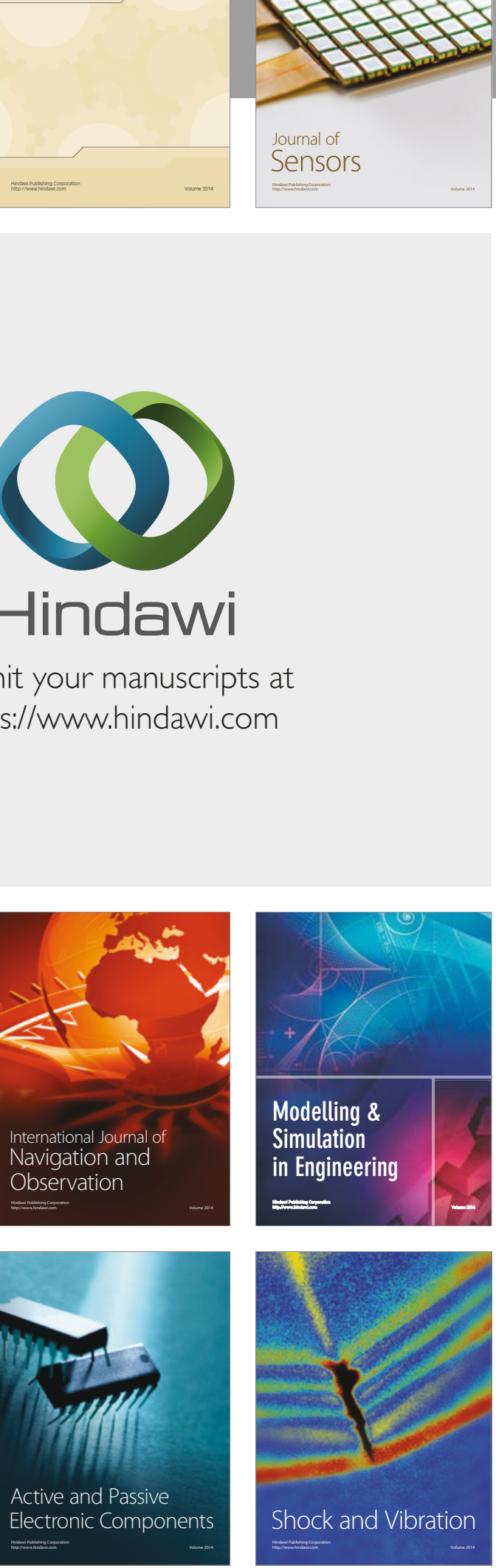
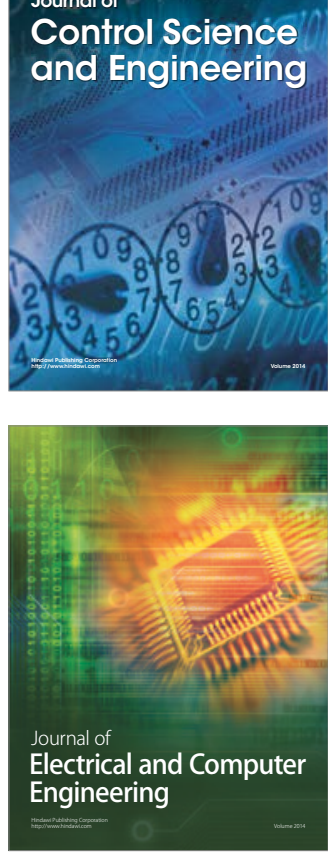

Distributed

Journal of

Control Science

and Engineering
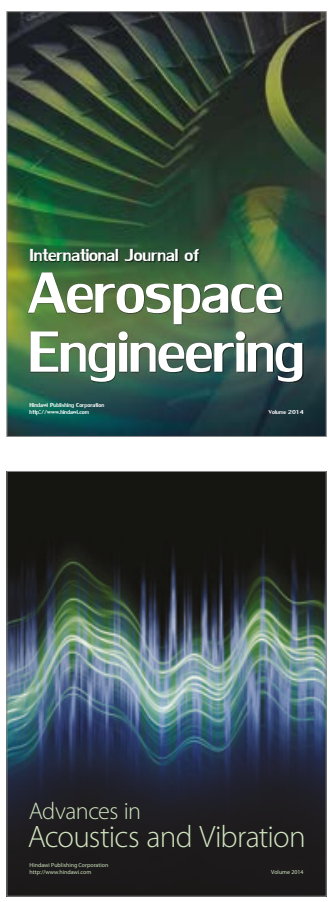

Sensor Networks 